\title{
Customer Reviews' Sentiments Analysis using Deep Learning
}

\author{
Kusum Mehta \\ Manav Rachna International Institute of Research and \\ Studies
}

\author{
Supriya P. Panda, PhD \\ Manav Rachna International Institute of Research and \\ Studies
}

\begin{abstract}
In this era of digitalization, Sentiment Analysis(SA) has become a necessity for progress and prosperity in marketing. Sentiment analysis has become a powerful way of knowing the opinions and thoughts of users. The viewpoint of the consumer, such as knowledge sharing would include a lot of useful experience, while one wrong idea will cost too much for the company.SA has many social media data-related problems, such as natural language interpretation, etc. Issue of theory and technique also affect the accuracy of detecting the polarity. There is a problem of text classification such as analysis of sentiments in document level, sentence level, feature based. Document level analysis is done by two approaches: supervised learning and unsupervised learning. Sentence level contains sentences containing opinions. Aspect based analysis have different attributes is performed in customer reviews. Product opinion is taken for knowing the sentiments. Opinions are compared and are extracted as a feature.SA is very important for business purpose because it gives the way for improving their operations and the products they offer. For improving business strategy it plays an important role. It provides many key benefits like impactful decisions, for finding relevant products, improving business strategy, beating competitions, tackling positive or negative issues affecting the product. To overcome such issues, deep learning processes are applied. The work focuses on two main tasks. Firstly, to extract sentiments present in data of social media of customers' reviews and secondly, to use the deeplearning process for the sentiments' extraction for customer reviews.
\end{abstract}

\section{Keywords}

Sentiment Analysis, Neural Networks, NLP, Deep Learning, Convolutional Neural Networks, Machine Learning

\section{INTRODUCTION}

The prime data sources are the frequent use of a web tool, social networking sites, blogging sites, and forms. It enables the user to share opinions, comments, feedback, and discussion on important topics. The demand for online shopping, marketing communication, etc. has grown rapidly due to the rise of the internet and access to its users. It has become meaningful to analyze public opinion to explain their activities and moods and the influence of other opinions. Hussein et al., discuss customer reviews that contain a large piece of information. There is, however, a need for data retrieval since it is buried in behaviors, perceptions, and emotions [1]. Receiving input from consumers will unexpectedly raise earnings. Such reports aid in the happiness of clients. Valuable information is shared by customers, which industries cannot ignore. The Sentiment Analysis tool and the analysis of data help in decision making. Emotions described as mood, behavior, actions, and mind state that influence the life are expressed through conversation. Emotion detection is primarily done by expressions like happiness, surprise, sadness, fear, and anger, etc. Written language is also an effective means of communicating ideas, knowledge, feelings, etc. Emotions are states of mind, which are expressed through words. The objective is the determination of the user's thoughts whether they are positive, negative, or neutral. Extraction is done at three levels namely Document Level, Sentence Level, and Feature Level.

There are three methods: Lexicon-Based, Machine Learning, and Hybrid. The first is further divided into Dictionary and Corpus-based..And Machine Learning-based technique is also divided into two models; the Traditional model and the Deep Learning model. The traditional model refers to classification such as Naïve Bayes, Support Vector Machines (SVM), and Maximum Entropy (ME). Hybrid approach is a combination of a lexicon-based and machine learning-based approach. Deep learning is also a process of machine learning by gaining importance in the area of research. It has been widely used as image datasets extract significant features of an image as shown in Figure 1.

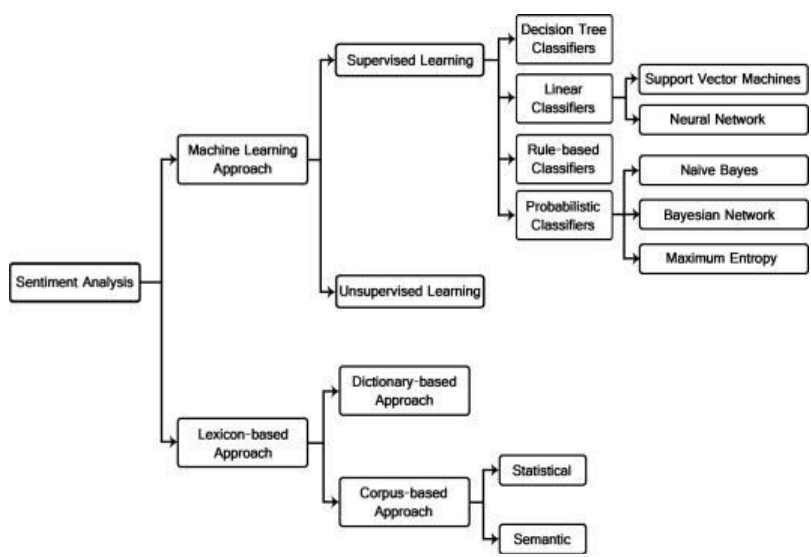

Figure 1:Picture of taxonomy of sentiment analysis Classification Methods of Sentiment Analysis

Work is focused on two main tasks. The first is to extract sentiments in customer reviews; Convolutional Neural Network(CNN) does all the extracting and describing of the feature. The Second Deep Learning Task is the extraction the sentiments for customer reviews. To optimize the classifier's parameters, Classification errors are minimized. CNN emphasizes words that affect from classification and parts of features that are of high thrust.

Strapparava et al. mention that sentiment analysis is quite beneficial in fields like health, business, and sports, to name a few [2]. In the business field, companies can know customers behavior for attracting new customers. Companies can improve their product and can improve marketing ways. By understanding the opinion, the recommender system may also 
become helpful. Jeong et al. proposed the technique of topic modeling and analyzed sentiments on customer-generated data[3]. Every person has different ways of expression. CNN was implemented in sequential order for the classification of emotions, which utilizes the feature of the sentence for the detection of emotions. For capturing feature of input, a trained word embedding technique is used as the first layer that changes words into a vector. For verification, features are passed to the attending model. Thus CNN takes these features, which are very useful as input. RNN, LSTM, and Random Forest Classifiers are used to compare results. There are mainly two objectives:

\section{To Extract sentiments by customer reviews.}

\section{To design a model of $\mathrm{CNN}$ for predicting results.}

\section{RELATED WORK}

Tang et al. proposed a deep learning approach, word embedding, extraction of opinions, etc. that focused to find emotional keywords. The comparison study was done on review data sets. When result was evaluated time was not taken into consideration[4].Yanarn et al. applied a basic theory and verified that emotional words represent a real process and category related to keywords [5]. Zhang et al. applied machine learning method for the analyzation of sentiments Datasets were used containing scripted dialogs and improvised dialogs .Only improvised data was used. Raw spectrogram was applied as an input[6]. Spoken words were used as a text feature and the TF-IDF approach was used for calculating weights of words. Sharef et.al. proposed sentiment analysis by deep learning method[7]. Liu et al. built a model to analyze emotions and classification was done in categories according to Ekman's model. Real world common sense knowledge was taken into account for classification which was very expensive[8]. Strapparava et al. proposed the same words having different senses to solve this problem, a set of effective words was made by using WORDNET. Lexical semantic approach cannot distinguish between different sense of same words[9]. Jain et al. proposed a Naïve Bayes Classifier for automatic emotion detection. Many hierarchal studies were used to classify emotion categories on a finegrained level[10]. Jain and Kumar classified emotions by machine learning approaches in multilingual data [11].]According to Jain and Kumar, 2017 buyer thoughts are helpful for buyers and sellers for improving market strategy[12].Mikolov et al. provided work for word embedding[13]. Paredes-Valverde et al. proposed a sentiment analysis for customer satisfaction by improving services[14]. Alharbi et al. proposed a method of extraction of features to know the behavior of users in which CNN was used[15]. Pham et al. analyzed travel reviews for extraction of sentiment [16]. Preethi et al. proposed a topic modeling technique for customer-generated data. For changing customer needs real-time monitoring tool was used [17]. Vateekul et al. applied polarity based sentiments methods for analyzing tweets [18]. Salas-Zarate et al.used aspect-based sentiment analysis was done. Senti-word net dictionary was used [19].Satapathy et al. analysesentiments on mental health data posted by patients[20]. Chorowski et al.recognized attention-based models for speech recognition [21]..Cireganet.al. proposed methods of deep learning process for classification of images and recognition of patterns [22].

.DaSilva et al. proposed ANN architectures and training processes using artificial neural networks [23]. Ghazi et al. proposed that for automatic classification of emotions two level classification was done. In the first level positive and negative emotion was classified and last level emotion was fine grained. Different levels of hierarchy are not used at different tasks [24].

Deep neural networks has brought revolutionary change in the field of natural language processing where data features is captured by many layers and gives an output...It provides understanding and capability of learning of feature ad helps in solving complications of artificial intelligence accurately.

\section{PROPOSED METHODOLOGY}

Two data sets are used for analysis; the first for movie reviews and the second for the Amazon company. These data sets include labeled review data with a score of 0 for negative sentiment and 1 for positive sentiment. The number of comments about a product has a significant effect on awareness, intentions, expectations, and behavior key for selling products respond to demand of customers at the proper time in the right locations. Each word for review is meaningful for decision and helpful for improving satisfactions. These words are full of slangs and misspellings due to many languages. Preprocessing is very necessary for making data normal. Data sets used for analysis were taken from Kaggle.

Labeling is based on the star rating. The one or two stars labeled negative, 3 as neutral, 4 , and 5 as positive .Many pages of company were observed for review and these were taken from website. The term used for observing are("Mobiles," Bed", "Chairs","Camera",Tab").The three catagoriesof product was made as shown in a table. Randomly 59000 reviews were extracted ,39500 were used to train classifier and 19500 were used for testing. Reviews of various products are collected as in Table1.

Table 1: Used Data Sets

\begin{tabular}{|l|l|}
\hline Search Data Sets & Reviews Numbers \\
\hline Sets for training & 39,500 \\
\hline Sets for testing & 19,500 \\
\hline Evaluation sets & \\
\hline Kaggle & $4,000,000$ \\
\hline Electronic Devices & 65000 \\
\hline Toys & 21613 \\
\hline Wooden furniture & 150,000 \\
\hline
\end{tabular}

\section{Data Preprocessing:}

Preprocessing is very necessary for cleaning raw data. The following methods are applied: Tag removal, filtration of symbols except for (A-Z) and numbers (0-9).

Feature Extraction: This process is done to convert text data into numerical data. Text words are separated one by one and are converted into unique integers. After tokenization, the whole sentence is represented by numerical data. For the classification of review, three machine learning approaches are used: Naïve Bayes' (Multimonial) ,SVM(Linear),and $\mathrm{CNN}$ with word embedding.

Data is randomly split into $75 \%$ training data and $25 \%$ testing data. The model created has four layers. Knowing the accuracy of classification following metrics was used. The prediction of sentiment analysis was done by star rating.

Basic steps used to train CNN model shown in Figure 2. 


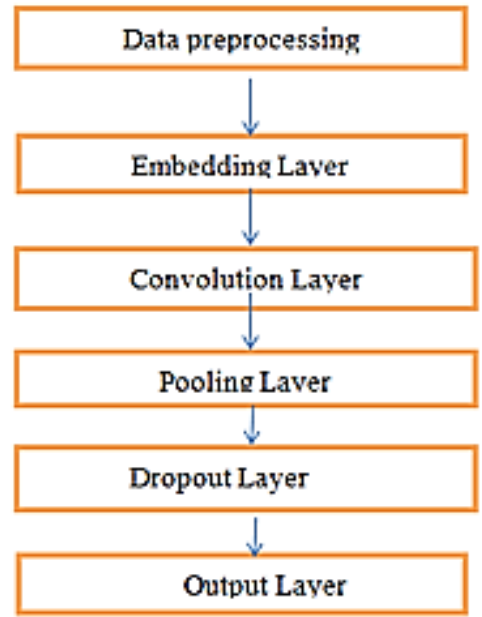

Figure 2:Basic steps used to train CNN model

The algorithm for predicting sentiment polarity is used to assess the efficiency of techniques and accordingly, classification is performed. Algorithm performance is tested by two methods: Word embedding and TF-IDF approach.Sentences are split into words individually and converted into a vector of real numbers called feature vectors. These feature vectors are used as input of this model.

\section{Embedding Layer}

CNN layer defined depends on a kernel of 3 sizes and 32 filters. Itmeans 32 different features on the first layer. The output of the first layer is $40 * 32$ neuron matrix. This result is fed to the second layer accordingly. The output matrix will measure $40 * 16$. The pooling layer is used after the $\mathrm{CNN}$ layer for the prevention of overfitting data. The output matrix has a neuron long dimension of $1 * 8$.

The output layer is a fully-connected layer with a sigmoid function that reduces the vector of height. F-score was derived from precision and recall. Different types of data sets with feature extraction are used. Performance stars' deep learning technique with word embedding is better than TFIDF.

Pair of feature vectors and tags that is positive, negative, neutral is fed into a machine-learning algorithm to generate a model, as shown in Figure 3.

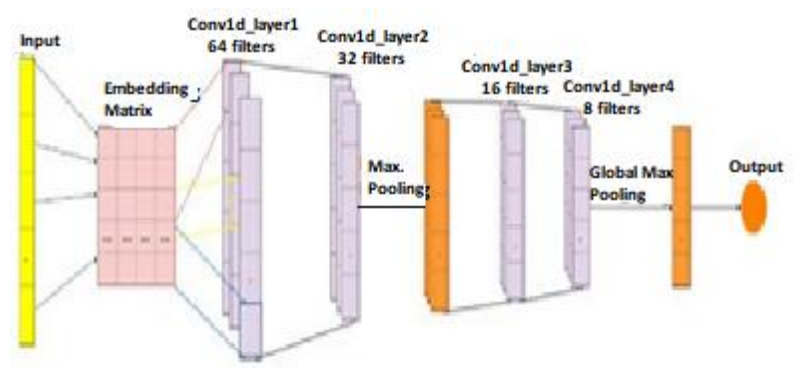

Figure 3: Model of CNN

\section{Algorithm applied before feeding into CNN}

1) Each sample tagged term is summed up.

2) For the formation of the training set, low-frequency samples are extracted.

3) The CNN model has been extracted. The first layer of CNN is fully connected to the second layer for future fusion.
4) Input all the training sets into the $\mathrm{CNN}$ channel and conduct training...Deriu et al proposed many convolutions and pooling layers are used for large data in CNN[25].Word sequence is given as an input word vector is used to train the CNN model.

For the evaluation process, 4-

million reviews of Kaggle data sets are collected.

Good performance giving classifiers are applied in reviews. These are done for different categories as shown in Table 2:

Table 2:Results of Evaluation Data
\begin{tabular}{|c|c|c|c|c|}
\hline Dataset & Accuracy & Precision & Recall & $\begin{array}{c}\text { F1- } \\
\text { score }\end{array}$ \\
\hline $\begin{array}{c}\text { 4MReview } \\
\text { set }\end{array}$ & 0.89 & 0.74 & 0.86 & 0.88 \\
\hline Toys & 0.90 & 0.98 & 0.9 & 0.94 \\
\hline $\begin{array}{c}\text { Electronic } \\
\text { Products }\end{array}$ & 0.89 & 0.97 & 0.9 & 0.93 \\
\hline $\begin{array}{c}\text { Wooden } \\
\text { Furniture }\end{array}$ & 0.91 & 0.97 & 0.92 & 0.94 \\
\hline
\end{tabular}

Table 3:Comparisons of different classifiers

\begin{tabular}{|c|c|c|c|c|}
\hline Classifiers & Accuracy & Precision & Recall & $\begin{array}{c}\text { F1- } \\
\text { score }\end{array}$ \\
\hline SVM & 0.85 & 0.85 & 0.85 & 0.85 \\
\hline NaïveBayes & 0.84 & 0.86 & 0.81 & 0.83 \\
\hline $\begin{array}{c}\text { CNN plus } \\
\text { word } \\
\text { embedding }\end{array}$ & 0.89 & 0.91 & 0.86 & 0.89 \\
\hline
\end{tabular}

\section{TF.DF WORD EMBEDDING}

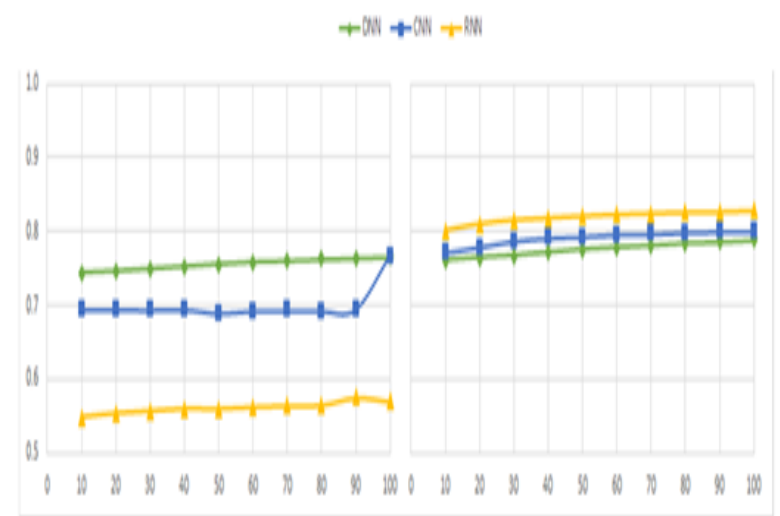

Figure 4 :TF-IDF and Word Embedding representation by graph

To know accuracy value these two approaches for different number of data sets are used. Precision, recall and F1 score value is calculated by this method with $\mathrm{CNN}$.

\section{Prediction of sentiments from text data}

The two approaches shown in figure 4 are applied for calculation of Precision, Recall and F1-score with CNN Model.

Prediction of sentiments from text data

Reviews ratings are numbered 1 to 5 , where 1 is for the worst and 5 is for the best. 
Import pandas as pd

Products=pd.read csv('amazon baby.csv')

Products.head()

For removing nan values data is cleaned

Len(products)-len(products.dropna())

Products $=$ products.dropna()

Every row data is checked.

New data is changed to string.

For $\mathrm{i}$ in range(0,len(products)-1):

If type(products.iloc[i]

['review'])!=str:

products.iloc[i]

['review'] $=$ strproducts.iloc[i]['review'])

The model is taught about positive and negative emotions when doing sentiment analysis. A ' 3 ' is neglected because it is neither good nor bad.

The written functions depends upon rating.It returns to 1 if the rating is 4 or more else written 0 . This function is applied to create new column.

At this time sentiment classifier is developed. Firstly, the training feature is prepared 'name' and 'review' are combined to form a single column. Firstly function is written, the feature is combined with a combination of both columns. A new column has created all features that contain strings. Here is the step by step process:

Input variable $\mathrm{x}$ and output variable $\mathrm{y} . \mathrm{X}$ is the features column and $\mathrm{y}$ is the sentiments column.

\section{$\mathrm{X}=$ products['all features'] \\ $\mathrm{Y}=$ products ['sentiment']}

Data sets are split into testing and training sets.Train_test _split function from sci_kit learn library. $80 \%$ is for training and $20 \%$ is for testing.

From sklearn.model_selection

Import train_test_split

X_train,X_test,y_train,y_test=train_test_split(X,y,randomstat $\mathrm{e}=0$ )

Import count vectorizer and tested and training data is fitted in it.

From

Sklearn.feature_extraction.textimportCountVectorizer

$\mathrm{cv}=$ CountVectorizer ()

ctmTr=cv.fit_transform(X_train)

X-test_dtm=cv.transform $\left(X \_t e s t\right)$

Trained model is used to predict sentiments for test data.

\section{CONCLUSION}

In this paper description of deep learning models and the related techniques are described to analyze sentiments of social media data; due to the urge for real-time rapid decision in digital business. .CNN model is very important for rapid decision making. Word embedding and the TF-IDF approach are combined with $\mathrm{CNN}$ to perform analysis of sentiments. Data sets of reviews with different protects have experimented with this approach. It is concluded that the accuracy of classification is improved by deep learning. Sentiment Polarity analysis is done by feature extraction. After comparing methods and doing experiments it has been found that CNN with word embedding is better than other classification techniques in customer reviews as shown by accuracy in Table 3. Future work will be focused on a hybrid approach.

\section{REFERENCES}

[1] Hussein, D.M.E.D.M., 2018. A survey on sentiment analysis challenges. Journal of King Saud UniversityEngineering Sciences, 30(4), pp.330-338.

[2] Strapparava, C. and Mihalcea, R., 2008, March.Learning to identify emotions in text.In Proceedings of the 2008 ACM symposium on Applied computing (pp. 1556-1560).

[3] Jeong, B., Yoon, J. and Lee, J.M., 2019. Social media mining for product planning: A product opportunity mining approach based on topic modeling and sentiment analysis. International Journal of Information Management, 48, pp.280-290.

[4] Tang, D., Qin, B. and Liu, T., 2015. Deep learning for sentiment analysis: successful approaches and future challenges. Wiley Interdisciplinary Reviews: Data Mining and Knowledge Discovery, 5(6), pp.292-303.

[5] Torao, Y., Naruki, S., Kaori, Y. and Masahiro, N., 1997. An emotion processing system based on fuzzy inference and subjective observations. Information sciences, 101(34), pp.217-247.

[6] Zhang, Y., Du, J., Wang, Z., Zhang, J. and Tu, Y., 2018, November. Attention based fully convolutional network for speech emotion recognition, In 2018 Asia-Pacific Signal and Information Processing Association Annual Summit and Conference (APSIPA ASC) (pp. 1771-1775). IEEE.

[7] Sharef, N.M., Zin, H.M. and Nadali, S., 2016.Overview and Future Opportunities of Sentiment Analysis Approaches for Big Data. J. Comput. Sci., 12(3), pp.153168.

[8] Liu, M., Li, S., Shan, S., Wang, R. and Chen, X., 2014, November. Deeply learning deformable facial action parts model for dynamic expression analysis,In Asian conference on computer vision ,pp. 143-157,Springer, Cham

[9] [9] Strapparava, C. and Valitutti, A., 2004, May. Wordnet affect: an affective extension of wordnet. In ,Lrec(Vol. 4, No. 1083-1086, p. 40)

[10] Jain, V.K., Kumar, S., Jain, N. andVerma, P., 2016, May. A novel approach to track public emotions related to epidemics in multilingual data.In 2nd International Conference and Youth School Information Technology and Nanotechnology (ITNT 2016),Russia ,pp. 883-889

[11] Jain, V.K., Kumar, S. and Fernandes,S.L.,2017. Extraction of emotions from multilingual text using intelligent text processing and computational linguistics. Journal of computational science, 21, pp.316-326.

[12] Jain, V.K. and Kumar, S., 2020.Predictive analysis of emotions for improving customer services. In Natural Language Processing: Concepts, Methodologies, Tools, and Applications (pp. 808-817). IGI Global.

[13] Mikolov, T., Chen, K., Corrado, G. and Dean, J., 2013. Efficient estimation of word representations in vector space. arXiv preprint arXiv:1301.3781. 
[14] Paredes-Valverde, M.A., Colomo- Palacios, R., SalasZárate, M.D.P. and Valencia-García, R., 2017. Sentiment analysis in Spanish for improvement of products and services: A deep learning approach. Scientific Programming, 2017

[15] Alharbi, A.S.M. and de Doncker, E.,2019. Twitter sentiment analysis with a deepneural network: An enhanced approach usinguser behavioral information. Cognitive Systems Research, 54, pp.50-61

[16] Pham, D.H. and Le, A.C., 2018. Learning multiple layers of knowledge representation for aspect based sentiment analysis. Data \& Knowledge Engineering, 114, pp.26-39

[17] Preethi, G., Krishna, P.V., Obaidat, M.S., Saritha, V. and Yenduri, S., 2017, July. Application of deep learning to sentiment analysis for recommender system on cloud. In 2017,International Conference on Computer, Information and Tecommunication Systems (CITS) (pp. 93-97). IEEE

[18] Vateekul, P. and Koomsubha, T., 2016, July. A study of sentiment analysis using deep learning techniques on Thai Twitter data. In 2016 13th International Joint Conference on Computer Science and Software Engineering (JCSSE) (pp. 1-6). IEEE

[19] Salas-Zárate,M.D.P., Medina-Moreira, J., Lagos-Ortiz, K., Luna-Aveiga, H., Rodriguez-Garcia, M.A. and Valencia-Garcia, R., 2017. Sentiment analysis on tweets about diabetes: an aspect-level approach. Computational and mathematical methods in medicine, 2017.
[20] Satapathy, R., Cambria, E. and Hussain, A., 2017. Sentiment Analysis in the Bio-Medical Domain. Springer: Berlin,Germa.ny.

[21] Chorowski, J.K., Bahdanau, D., Serdyuk, D., Cho, K. andBengio, Y., 2015. Attention-based models for speech recognition.In Advances in neural information processing systems(pp 577-585)

[22] Ciregan, D., Meier, U. and Schmidhuber, J., 2012, June. Multi-column deep neural networks for image classification.In 2012 IEEE conference on computer vision and pattern recognition (pp. 3642-3649).IEEE.

[23] Da Silva, I.N., Spatti, D.H., Flauzino, R.A., Liboni, L.H.B. and dos Reis Alves, S.F., 2017.Artificial neural network architecturesture and training processes.In Artificial neural networks (pp.21- 21-28).Springer, Cham.

[24] Ghazi, D., Inkpen, D. and Szpakowicz, S., 2010, June. Hierarchical versus flat classification of emotionsin intext.In Proceedings of the NAACL HLT 2010 workshop on computational approaches to analysis and generation of emotion in text (pp. 140-146)

[25] Deriu, J.M. and Cieliebak, M., 2016. Sentiment analysis usingsing convolutional neural networks with multi-task training and distant supervision on italian tweets. In Fifth Evaluation Campaign of Natural Language Processing and Speech Tools for Italian,Napoli,Italy 5-7 December 2016. Italian Journal of Computational Linguistics. 